\title{
Study of $B^{0} \rightarrow \pi^{0} \pi^{0}, B^{ \pm} \rightarrow \pi^{ \pm} \pi^{0}$, and $B^{ \pm} \rightarrow K^{ \pm} \pi^{0}$ decays, and isospin analysis of $B \rightarrow \pi \pi$ decays
}

B. Aubert, ${ }^{1}$ M. Bona, ${ }^{1}$ D. Boutigny, ${ }^{1}$ Y. Karyotakis, ${ }^{1}$ J. P. Lees, ${ }^{1}$ V. Poireau, ${ }^{1}$ X. Prudent,${ }^{1}$ V. Tisserand,${ }^{1}$ A. Zghiche,${ }^{1}$ J. Garra Tico, ${ }^{2}$ E. Grauges ${ }^{2}$ L. Lopez,${ }^{3}$ A. Palano, ${ }^{3}$ M. Pappagallo, ${ }^{3}$ G. Eigen, ${ }^{4}$ B. Stugu, ${ }^{4}$ L. Sun,${ }^{4}$ G. S. Abrams,${ }^{5}$ M. Battaglia, ${ }^{5}$ D. N. Brown, ${ }^{5}$ J. Button-Shafer, ${ }^{5}$ R. N. Cahn, ${ }^{5}$ Y. Groysman, ${ }^{5}$ R. G. Jacobsen, ${ }^{5}$ J. A. Kadyk, ${ }^{5}$ L. T. Kerth, ${ }^{5}$ Yu. G. Kolomensky, ${ }^{5}$ G. Kukartsev, ${ }^{5}$ D. Lopes Pegna, ${ }^{5}$ G. Lynch, ${ }^{5}$ L. M. Mir, ${ }^{5}$ T. J. Orimoto, ${ }^{5}$ I. L. Osipenkov, ${ }^{5}$ M. T. Ronan, ${ }^{5, *}$ K. Tackmann, ${ }^{5}$ T. Tanabe, ${ }^{5}$ W. A. Wenzel, ${ }^{5}$ P. del Amo Sanchez, ${ }^{6}$ C. M. Hawkes, ${ }^{6}$ A. T. Watson, ${ }^{6}$ T. Held, ${ }^{7}$ H. Koch, ${ }^{7}$ M. Pelizaeus, ${ }^{7}$ T. Schroeder, ${ }^{7}$ M. Steinke, ${ }^{7}$ D. Walker, ${ }^{8}$ D. J. Asgeirsson, ${ }^{9}$ T. Cuhadar-Donszelmann, ${ }^{9}$ B. G. Fulsom, ${ }^{9}$ C. Hearty, ${ }^{9}$ T. S. Mattison, ${ }^{9}$ J. A. McKenna, ${ }^{9}$ A. Khan, ${ }^{10}$ M. Saleem, ${ }^{10}$ L. Teodorescu, ${ }^{10}$ V. E. Blinov, ${ }^{11}$ A. D. Bukin, ${ }^{11}$ V. P. Druzhinin, ${ }^{11}$ V. B. Golubev, ${ }^{11}$ A. P. Onuchin, ${ }^{11}$ S. I. Serednyakov, ${ }^{11}$ Yu. I. Skovpen, ${ }^{11}$ E. P. Solodov, ${ }^{11}$ K. Yu. Todyshev, ${ }^{11}$ M. Bondioli, ${ }^{12}$ S. Curry, ${ }^{12}$ I. Eschrich, ${ }^{12}$ D. Kirkby, ${ }^{12}$ A. J. Lankford,,${ }^{12}$ P. Lund, ${ }^{12}$ M. Mandelkern, ${ }^{12}$ E. C. Martin, ${ }^{12}$ D. P. Stoker, ${ }^{12}$ S. Abachi, ${ }^{13}$ C. Buchanan, ${ }^{13}$ S. D. Foulkes,${ }^{14}$ J. W. Gary, ${ }^{14}$ F. Liu, ${ }^{14}$ O. Long, ${ }^{14}$ B. C. Shen,${ }^{14}$ L. Zhang, ${ }^{14}$ H. P. Paar, ${ }^{15}$ S. Rahatlou, ${ }^{15}$ V. Sharma, ${ }^{15}$ J. W. Berryhill, ${ }^{16}$ C. Campagnari, ${ }^{16}$ A. Cunha, ${ }^{16}$ B. Dahmes, ${ }^{16}$

T. M. Hong, ${ }^{16}$ D. Kovalskyi, ${ }^{16}$ J. D. Richman, ${ }^{16}$ T. W. Beck, ${ }^{17}$ A. M. Eisner, ${ }^{17}$ C. J. Flacco, ${ }^{17}$ C. A. Heusch, ${ }^{17}$

J. Kroseberg, ${ }^{17}$ W. S. Lockman, ${ }^{17}$ T. Schalk, ${ }^{17}$ B. A. Schumm, ${ }^{17}$ A. Seiden, ${ }^{17}$ M. G. Wilson, ${ }^{17}$ L. O. Winstrom, ${ }^{17}$ E. Chen, ${ }^{18}$ C. H. Cheng, ${ }^{18}$ F. Fang, ${ }^{18}$ D. G. Hitlin, ${ }^{18}$ I. Narsky, ${ }^{18}$ T. Piatenko, ${ }^{18}$ F. C. Porter, ${ }^{18}$ R. Andreassen, ${ }^{19}$ G. Mancinelli, ${ }^{19}$ B. T. Meadows, ${ }^{19}$ K. Mishra, ${ }^{19}$ M. D. Sokoloff, ${ }^{19}$ F. Blanc, ${ }^{20}$ P. C. Bloom, ${ }^{20}$ S. Chen,${ }^{20}$ W. T. Ford,${ }^{20}$ J. F. Hirschauer, ${ }^{20}$ A. Kreisel, ${ }^{20}$ M. Nagel, ${ }^{20}$ U. Nauenberg, ${ }^{20}$ A. Olivas, ${ }^{20}$ J. G. Smith,${ }^{20}$ K. A. Ulmer, ${ }^{20}$ S. R. Wagner, ${ }^{20}$ J. Zhang, ${ }^{20}$ A. M. Gabareen, ${ }^{21}$ A. Soffer, ${ }^{21, \dagger}$ W. H. Toki ${ }^{21}$ R. J. Wilson, ${ }^{21}$ F. Winklmeier,${ }^{21}$ D. D. Altenburg, ${ }^{22}$ E. Feltresi, ${ }^{22}$ A. Hauke, ${ }^{22}$ H. Jasper, ${ }^{22}$ J. Merkel, ${ }^{22}$ A. Petzold, ${ }^{22}$ B. Spaan, ${ }^{22}$ K. Wacker, ${ }^{22}$ V. Klose, ${ }^{23}$ M. J. Kobel, ${ }^{23}$ H. M. Lacker, ${ }^{23}$ W. F. Mader, ${ }^{23}$ R. Nogowski, ${ }^{23}$ J. Schubert, ${ }^{23}$ K. R. Schubert, ${ }^{23}$ R. Schwierz, ${ }^{23}$ J. E. Sundermann, ${ }^{23}$ A. Volk, ${ }^{23}$

D. Bernard, ${ }^{24}$ G. R. Bonneaud, ${ }^{24}$ E. Latour, ${ }^{24}$ V. Lombardo,${ }^{24}$ Ch. Thiebaux, ${ }^{24}$ M. Verderi, ${ }^{24}$ P. J. Clark,,${ }^{25}$ W. Gradl, ${ }^{25}$ F. Muheim ${ }^{25}$ S. Playfer ${ }^{25}$ A. I. Robertson, ${ }^{25}$ J. E. Watson, ${ }^{25}$ Y. Xie,${ }^{25}$ M. Andreotti, ${ }^{26}$ D. Bettoni, ${ }^{26}$ C. Bozzi, ${ }^{26}$ R. Calabrese, ${ }^{26}$ A. Cecchi, ${ }^{26}$ G. Cibinetto ${ }^{26}$ P. Franchini, ${ }^{26}$ E. Luppi, ${ }^{26}$ M. Negrini, ${ }^{26}$ A. Petrella,${ }^{26}$ L. Piemontese, ${ }^{26}$

E. Prencipe ${ }^{26}$ V. Santoro, ${ }^{26}$ F. Anulli, ${ }^{27}$ R. Baldini-Ferroli, ${ }^{27}$ A. Calcaterra,${ }^{27}$ R. de Sangro, ${ }^{27}$ G. Finocchiaro, ${ }^{27}$ S. Pacetti, ${ }^{27}$ P. Patteri, ${ }^{27}$ I. M. Peruzzi, ${ }^{27, \sharp}$ M. Piccolo, ${ }^{27}$ M. Rama, ${ }^{27}$ A. Zallo, ${ }^{27}$ A. Buzzo,${ }^{28}$ R. Contri, ${ }^{28}$ M. Lo Vetere,${ }^{28}$ M. M. Macri, ${ }^{28}$ M. R. Monge, ${ }^{28}$ S. Passaggio, ${ }^{28}$ C. Patrignani, ${ }^{28}$ E. Robutti, ${ }^{28}$ A. Santroni, ${ }^{28}$ S. Tosi, ${ }^{28}$

K. S. Chaisanguanthum, ${ }^{29}$ M. Morii, ${ }^{29}$ J. Wu ${ }^{29}$ R. S. Dubitzky, ${ }^{30}$ J. Marks,${ }^{30}$ S. Schenk, ${ }^{30}$ U. Uwer, ${ }^{30}$ D. J. Bard, ${ }^{31}$

P. D. Dauncey, ${ }^{31}$ R. L. Flack, ${ }^{31}$ J. A. Nash, ${ }^{31}$ W. Panduro Vazquez, ${ }^{31}$ M. Tibbetts, ${ }^{31}$ P. K. Behera, ${ }^{32}$ X. Chai, ${ }^{32}$

M. J. Charles, ${ }^{32}$ U. Mallik, ${ }^{32}$ V. Ziegler, ${ }^{32}$ J. Cochran, ${ }^{33}$ H. B. Crawley, ${ }^{33}$ L. Dong, ${ }^{33}$ V. Eyges, ${ }^{33}$ W. T. Meyer, ${ }^{33}$ S. Prell,${ }^{33}$

E. I. Rosenberg, ${ }^{33}$ A. E. Rubin, ${ }^{33}$ Y. Y. Gao, ${ }^{34}$ A. V. Gritsan, ${ }^{34}$ Z. J. Guo, ${ }^{34}$ C. K. Lae,${ }^{34}$ A. G. Denig, ${ }^{35}$ M. Fritsch, ${ }^{35}$ G. Schott, ${ }^{35}$ N. Arnaud, ${ }^{36}$ J. Béquilleux, ${ }^{36}$ A. D’ Orazio, ${ }^{36}$ M. Davier, ${ }^{36}$ G. Grosdidier, ${ }^{36}$ A. Höcker, ${ }^{36}$ V. Lepeltier, ${ }^{36}$

F. Le Diberder, ${ }^{36}$ A. M. Lutz, ${ }^{36}$ S. Pruvot, ${ }^{36}$ S. Rodier, ${ }^{36}$ P. Roudeau, ${ }^{36}$ M. H. Schune, ${ }^{36}$ J. Serrano, ${ }^{36}$ V. Sordini, ${ }^{36}$ A. Stocchi, ${ }^{36}$ W. F. Wang, ${ }^{36}$ G. Wormser, ${ }^{36}$ D. J. Lange, ${ }^{37}$ D. M. Wright, ${ }^{37}$ I. Bingham, ${ }^{38}$ C. A. Chavez, ${ }^{38}$ I. J. Forster, ${ }^{38}$

J. R. Fry, ${ }^{38}$ E. Gabathuler, ${ }^{38}$ R. Gamet, ${ }^{38}$ D. E. Hutchcroft, ${ }^{38}$ D. J. Payne, ${ }^{38}$ K. C. Schofield, ${ }^{38}$ C. Touramanis, ${ }^{38}$ A. J. Bevan, ${ }^{39}$ K. A. George, ${ }^{39}$ F. Di Lodovico, ${ }^{39}$ W. Menges,${ }^{39}$ R. Sacco, ${ }^{39}$ G. Cowan, ${ }^{40}$ H. U. Flaecher, ${ }^{40}$ D. A. Hopkins, ${ }^{40}$ S. Paramesvaran, ${ }^{40}$ F. Salvatore, ${ }^{40}$ A. C. Wren,${ }^{40}$ D. N. Brown, ${ }^{41}$ C. L. Davis, ${ }^{41}$ J. Allison, ${ }^{42}$ N. R. Barlow, ${ }^{42}$ R. J. Barlow, ${ }^{42}$ Y. M. Chia, ${ }^{42}$ C. L. Edgar, ${ }^{42}$ G. D. Lafferty, ${ }^{42}$ T. J. West, ${ }^{42}$ J. I. Yi, ${ }^{42}$ J. Anderson, ${ }^{43}$ C. Chen, ${ }^{43}$ A. Jawahery, ${ }^{43}$

D. A. Roberts, ${ }^{43}$ G. Simi, ${ }^{43}$ J. M. Tuggle, ${ }^{43}$ G. Blaylock, ${ }^{44}$ C. Dallapiccola, ${ }^{44}$ S. S. Hertzbach, ${ }^{44}$ X. Li,${ }^{44}$ T. B. Moore ${ }^{44}$ E. Salvati, ${ }^{44}$ S. Saremi, ${ }^{44}$ R. Cowan, ${ }^{45}$ D. Dujmic, ${ }^{45}$ P. H. Fisher,${ }^{45}$ K. Koeneke ${ }^{45}$ G. Sciolla,${ }^{45}$ S. J. Sekula ${ }^{45}$ M. Spitznagel, ${ }^{45}$ F. Taylor, ${ }^{45}$ R. K. Yamamoto, ${ }^{45}$ M. Zhao, ${ }^{45}$ Y. Zheng,${ }^{45}$ S. E. Mclachlin, ${ }^{46, *}$ P. M. Patel, ${ }^{46}$ S. H. Robertson, ${ }^{46}$ A. Lazzaro, ${ }^{47}$ F. Palombo, ${ }^{47}$ J. M. Bauer, ${ }^{48}$ L. Cremaldi, ${ }^{48}$ V. Eschenburg, ${ }^{48}$ R. Godang, ${ }^{48}$ R. Kroeger,${ }^{48}$ D. A. Sanders, ${ }^{48}$ D. J. Summers, ${ }^{48}$ H. W. Zhao, ${ }^{48}$ S. Brunet, ${ }^{49}$ D. Côté, ${ }^{49}$ M. Simard,${ }^{49}$ P. Taras, ${ }^{49}$ F. B. Viaud, ${ }^{49}$ H. Nicholson, ${ }^{50}$ G. De Nardo, ${ }^{51}$ F. Fabozzi,${ }^{51,}$ L. Lista, ${ }^{51}$ D. Monorchio, ${ }^{51}$ C. Sciacca, ${ }^{51}$ M. A. Baak, ${ }^{52}$ G. Raven,${ }^{52}$ H. L. Snoek ${ }^{52}$ C. P. Jessop, ${ }^{53}$ K. J. Knoepfel, ${ }^{53}$ J. M. LoSecco, ${ }^{53}$ G. Benelli, ${ }^{54}$ L. A. Corwin,${ }^{54}$ K. Honscheid ${ }^{54}$ H. Kagan, ${ }^{54}$ R. Kass, ${ }^{54}$ J. P. Morris ${ }^{54}$ A. M. Rahimi, ${ }^{54}$ J. J. Regensburger, ${ }^{54}$ Q. K. Wong, ${ }^{54}$ N. L. Blount, ${ }^{55}$ J. Brau, ${ }^{55}$ R. Frey, ${ }^{55}$ O. Igonkina, ${ }^{55}$ J. A. Kolb, ${ }^{55}$ M. Lu, ${ }^{55}$ R. Rahmat, ${ }^{55}$ N. B. Sinev, ${ }^{55}$ D. Strom,${ }^{55}$ J. Strube,${ }^{55}$ E. Torrence, ${ }^{55}$ N. Gagliardi ${ }^{56}$ A. Gaz,${ }^{56}$ M. Margoni, ${ }^{56}$ M. Morandin,,${ }^{56}$ A. Pompili, ${ }^{56}$ M. Posocco, ${ }^{56}$ M. Rotondo,${ }^{56}$ F. Simonetto, ${ }^{56}$ R. Stroili, ${ }^{56}$ C. Voci, ${ }^{56}$ E. Ben-Haim,${ }^{57}$ H. Briand,${ }^{57}$ G. Calderini, ${ }^{57}$ J. Chauveau, ${ }^{57}$ P. David,${ }^{57}$ L. Del Buono, ${ }^{57}$ Ch. de la Vaissière,${ }^{57}$ O. Hamon, ${ }^{57} \mathrm{Ph}$. Leruste, ${ }^{57}$ J. Malclès, ${ }^{57}$ J. Ocariz, ${ }^{57}$ A. Perez, ${ }^{57}$ J. Prendki, ${ }^{57}$ L. Gladney, ${ }^{58}$ M. Biasini, ${ }^{59}$ R. Covarelli, ${ }^{59}$ 
E. Manoni, ${ }^{59}$ C. Angelini ${ }^{60}$ G. Batignani,${ }^{60}$ S. Bettarini, ${ }^{60}$ M. Carpinelli, ${ }^{60}$ R. Cenci, ${ }^{60}$ A. Cervelli,${ }^{60}$ F. Forti, ${ }^{60}$ M. A. Giorgi, ${ }^{60}$ A. Lusiani, ${ }^{60}$ G. Marchiori, ${ }^{60}$ M. A. Mazur, ${ }^{60}$ M. Morganti,${ }^{60}$ N. Neri, ${ }^{60}$ E. Paoloni,${ }^{60}$ G. Rizzo, ${ }^{60}$ J. J. Walsh, ${ }^{60}$ M. Haire, ${ }^{61}$ J. Biesiada,${ }^{62}$ P. Elmer ${ }^{62}$ Y. P. Lau, ${ }^{62}$ C. Lu, ${ }^{62}$ J. Olsen, ${ }^{62}$ A. J. S. Smith, ${ }^{62}$ A. V. Telnov, ${ }^{62}$ E. Baracchini, ${ }^{63}$ F. Bellini, ${ }^{63}$ G. Cavoto, ${ }^{63}$ D. del Re, ${ }^{63}$ E. Di Marco,${ }^{63}$ R. Faccini, ${ }^{63}$ F. Ferrarotto, ${ }^{63}$ F. Ferroni, ${ }^{63}$ M. Gaspero, ${ }^{63}$ P. D. Jackson, ${ }^{63}$ L. Li Gioi, ${ }^{63}$ M. A. Mazzoni, ${ }^{63}$ S. Morganti, ${ }^{63}$ G. Piredda, ${ }^{63}$ F. Polci, ${ }^{63}$ F. Renga, ${ }^{63}$ C. Voena, ${ }^{63}$ M. Ebert, ${ }^{64}$ T. Hartmann, ${ }^{64}$ H. Schröder, ${ }^{64}$ R. Waldi, ${ }^{64}$ T. Adye, ${ }^{65}$ G. Castelli, ${ }^{65}$ B. Franek,${ }^{65}$ E. O. Olaiya ${ }^{65}$ S. Ricciardi, ${ }^{65}$ W. Roethel, ${ }^{65}$ F. F. Wilson, ${ }^{65}$ S. Emery, ${ }^{66}$ M. Escalier, ${ }^{66}$ A. Gaidot, ${ }^{66}$ S. F. Ganzhur, ${ }^{66}$ G. Hamel de Monchenault, ${ }^{66}$ W. Kozanecki, ${ }^{66}$ G. Vasseur, ${ }^{66}$ Ch. Yèche, ${ }^{66}$ M. Zito ${ }^{66}$ X. R. Chen,${ }^{67}$ H. Liu, ${ }^{67}$ W. Park,${ }^{67}$ M. V. Purohit, ${ }^{67}$ J. R. Wilson, ${ }^{67}$ M. T. Allen,${ }^{68}$ D. Aston, ${ }^{68}$ R. Bartoldus, ${ }^{68}$ P. Bechtle,${ }^{68}$ N. Berger, ${ }^{68}$ R. Claus, ${ }^{68}$ J. P. Coleman, ${ }^{68}$ M. R. Convery ${ }^{68}$ J. C. Dingfelder, ${ }^{68}$ J. Dorfan, ${ }^{68}$ G. P. Dubois-Felsmann, ${ }^{68}$ W. Dunwoodie, ${ }^{68}$ R. C. Field ${ }^{68}$ T. Glanzman, ${ }^{68}$ S. J. Gowdy, ${ }^{68}$ M. T. Graham, ${ }^{68}$ P. Grenier, ${ }^{68}$ C. Hast ${ }^{68}$ T. Hryn'ova, ${ }^{68}$ W. R. Innes, ${ }^{68}$ J. Kaminski, ${ }^{68}$ M. H. Kelsey, ${ }^{68}$ H. Kim, ${ }^{68}$ P. Kim, ${ }^{68}$ M. L. Kocian, ${ }^{68}$ D. W. G. S. Leith,${ }^{68}$ S. Li ${ }^{68}$ S. Luitz, ${ }^{68}$ V. Luth, ${ }^{68}$ H. L. Lynch, ${ }^{68}$ D. B. MacFarlane, ${ }^{68}$ H. Marsiske, ${ }^{68}$ R. Messner, ${ }^{68}$ D. R. Muller, ${ }^{68}$ C. P. O'Grady ${ }^{68}$ I. Ofte, ${ }^{68}$ A. Perazzo, ${ }^{68}$ M. Perl, ${ }^{68}$

T. Pulliam, ${ }^{68}$ B. N. Ratcliff, ${ }^{68}$ A. Roodman, ${ }^{68}$ A. A. Salnikov, ${ }^{68}$ R. H. Schindler, ${ }^{68}$ J. Schwiening, ${ }^{68}$ A. Snyder, ${ }^{68}$

J. Stelzer, ${ }^{68}$ D. Su, ${ }^{68}$ M. K. Sullivan, ${ }^{68}$ K. Suzuki, ${ }^{68}$ S. K. Swain, ${ }^{68}$ J. M. Thompson, ${ }^{68}$ J. Va'vra, ${ }^{68}$ N. van Bakel, ${ }^{68}$ A. P. Wagner, ${ }^{68}$ M. Weaver, ${ }^{68}$ W. J. Wisniewski, ${ }^{68}$ M. Wittgen, ${ }^{68}$ D. H. Wright, ${ }^{68}$ A. K. Yarritu ${ }^{68}$ K. Yi,${ }^{68}$ C. C. Young, ${ }^{68}$ P. R. Burchat, ${ }^{69}$ A. J. Edwards, ${ }^{69}$ S. A. Majewski, ${ }^{69}$ B. A. Petersen, ${ }^{69}$ L. Wilden, ${ }^{69}$ S. Ahmed,${ }^{70}$ M. S. Alam, ${ }^{70}$ R. Bula, ${ }^{70}$ J. A. Ernst, ${ }^{70}$ V. Jain, ${ }^{70}$ B. Pan, ${ }^{70}$ M. A. Saeed, ${ }^{70}$ F. R. Wappler, ${ }^{70}$ S. B. Zain, ${ }^{70}$ M. Krishnamurthy, ${ }^{71}$ S. M. Spanier, ${ }^{71}$ R. Eckmann, ${ }^{72}$ J. L. Ritchie, ${ }^{72}$ A. M. Ruland, ${ }^{72}$ C. J. Schilling, ${ }^{72}$ R. F. Schwitters, ${ }^{72}$ J. M. Izen, ${ }^{73}$ X. C. Lou, ${ }^{73}$ S. Ye, ${ }^{73}$ F. Bianchi, ${ }^{74}$ F. Gallo, ${ }^{74}$ D. Gamba,${ }^{74}$ M. Pelliccioni,${ }^{74}$ M. Bomben, ${ }^{75}$ L. Bosisio, ${ }^{75}$ C. Cartaro, ${ }^{75}$ F. Cossutti, ${ }^{75}$ G. Della Ricca ${ }^{75}$ L. Lanceri, ${ }^{75}$ L. Vitale, ${ }^{75}$ V. Azzolini,${ }^{76}$ N. Lopez-March,${ }^{76}$ F. Martinez-Vidal,${ }^{76, \|}$ D. A. Milanes, ${ }^{76}$ A. Oyanguren, ${ }^{76}$ J. Albert, ${ }^{77}$ Sw. Banerjee,${ }^{77}$ B. Bhuyan, ${ }^{77}$ K. Hamano,${ }^{77}$ R. Kowalewski, ${ }^{77}$ I. M. Nugent,${ }^{77}$ J. M. Roney,${ }^{77}$ R. J. Sobie, ${ }^{77}$ P. F. Harrison,${ }^{78}$ J. Ilic, ${ }^{78}$ T. E. Latham,${ }^{78}$ G. B. Mohanty, ${ }^{78}$ H. R. Band,${ }^{79}$ X. Chen,${ }^{79}$ S. Dasu ${ }^{79}$ K. T. Flood ${ }^{79}$ J. J. Hollar, ${ }^{79}$ P. E. Kutter, ${ }^{79}$ Y. Pan, ${ }^{79}$ M. Pierini,,${ }^{79}$ R. Prepost, ${ }^{79}$ S. L. Wu,${ }^{79}$ and H. Neal ${ }^{80}$

(BABAR Collaboration)

\footnotetext{
${ }^{1}$ Laboratoire de Physique des Particules, IN2P3/CNRS et Université de Savoie, F-74941 Annecy-Le-Vieux, France

${ }^{2}$ Universitat de Barcelona, Facultat de Fisica, Departament ECM, E-08028 Barcelona, Spain

${ }^{3}$ Università di Bari, Dipartimento di Fisica and INFN, I-70126 Bari, Italy

${ }^{4}$ University of Bergen, Institute of Physics, N-5007 Bergen, Norway

${ }^{5}$ Lawrence Berkeley National Laboratory and University of California, Berkeley, California 94720, USA

${ }^{6}$ University of Birmingham, Birmingham, B15 2TT, United Kingdom

${ }^{7}$ Ruhr Universität Bochum, Institut für Experimentalphysik 1, D-44780 Bochum, Germany

${ }^{8}$ University of Bristol, Bristol BS8 1TL, United Kingdom

${ }^{9}$ University of British Columbia, Vancouver, British Columbia, Canada V6T 1Z1

${ }^{10}$ Brunel University, Uxbridge, Middlesex UB8 3PH, United Kingdom

${ }^{11}$ Budker Institute of Nuclear Physics, Novosibirsk 630090, Russia

${ }^{12}$ University of California at Irvine, Irvine, California 92697, USA

${ }^{13}$ University of California at Los Angeles, Los Angeles, California 90024, USA

${ }^{14}$ University of California at Riverside, Riverside, California 92521, USA

${ }^{15}$ University of California at San Diego, La Jolla, California 92093, USA

${ }^{16}$ University of California at Santa Barbara, Santa Barbara, California 93106, USA

${ }^{17}$ University of California at Santa Cruz, Institute for Particle Physics, Santa Cruz, California 95064, USA

${ }^{18}$ California Institute of Technology, Pasadena, California 91125, USA

${ }^{19}$ University of Cincinnati, Cincinnati, Ohio 45221, USA

${ }^{20}$ University of Colorado, Boulder, Colorado 80309, USA

${ }^{21}$ Colorado State University, Fort Collins, Colorado 80523, USA

${ }^{22}$ Universität Dortmund, Institut für Physik, D-44221 Dortmund, Germany

${ }^{23}$ Technische Universität Dresden, Institut für Kern- und Teilchenphysik, D-01062 Dresden, Germany

${ }^{24}$ Laboratoire Leprince-Ringuet, CNRS/IN2P3, Ecole Polytechnique, F-91128 Palaiseau, France

${ }^{25}$ University of Edinburgh, Edinburgh EH9 3JZ, United Kingdom

${ }^{26}$ Università di Ferrara, Dipartimento di Fisica and INFN, I-44100 Ferrara, Italy

${ }^{27}$ Laboratori Nazionali di Frascati dell'INFN, I-00044 Frascati, Italy

${ }^{28}$ Università di Genova, Dipartimento di Fisica and INFN, I-16146 Genova, Italy

${ }^{29}$ Harvard University, Cambridge, Massachusetts 02138, USA
} 
${ }^{30}$ Universität Heidelberg, Physikalisches Institut, Philosophenweg 12, D-69120 Heidelberg, Germany

${ }^{31}$ Imperial College London, London, SW7 2AZ, United Kingdom

${ }^{32}$ University of Iowa, Iowa City, Iowa 52242, USA

${ }^{33}$ Iowa State University, Ames, Iowa 50011-3160, USA

${ }^{34}$ Johns Hopkins University, Baltimore, Maryland 21218, USA

${ }^{35}$ Universität Karlsruhe, Institut für Experimentelle Kernphysik, D-76021 Karlsruhe, Germany

${ }^{36}$ Laboratoire de l'Accélérateur Linéaire, IN2P3/CNRS et Université Paris-Sud 11, Centre Scientifique d'Orsay, BP 34, F-91898 ORSAY Cedex, France

${ }^{37}$ Lawrence Livermore National Laboratory, Livermore, California 94550, USA

${ }^{38}$ University of Liverpool, Liverpool L69 7ZE, United Kingdom

${ }^{39}$ Queen Mary, University of London, E1 4NS, United Kingdom

${ }^{40}$ University of London, Royal Holloway and Bedford New College, Egham, Surrey TW20 OEX, United Kingdom

${ }^{41}$ University of Louisville, Louisville, Kentucky 40292, USA

${ }^{42}$ University of Manchester, Manchester M13 9PL, United Kingdom

${ }^{43}$ University of Maryland, College Park, Maryland 20742, USA

${ }^{44}$ University of Massachusetts, Amherst, Massachusetts 01003, USA

${ }^{45}$ Massachusetts Institute of Technology, Laboratory for Nuclear Science, Cambridge, Massachusetts 02139, USA

${ }^{46}$ McGill University, Montréal, Québec, Canada H3A $2 T 8$

${ }^{47}$ Università di Milano, Dipartimento di Fisica and INFN, I-20133 Milano, Italy

${ }^{48}$ University of Mississippi, University, Mississippi 38677, USA

${ }^{49}$ Université de Montréal, Physique des Particules, Montréal, Québec, Canada H3C 3J7

${ }^{50}$ Mount Holyoke College, South Hadley, Massachusetts 01075, USA

${ }^{51}$ Università di Napoli Federico II, Dipartimento di Scienze Fisiche and INFN, I-80126, Napoli, Italy

${ }^{52}$ NIKHEF, National Institute for Nuclear Physics and High Energy Physics, NL-1009 DB Amsterdam, The Netherlands

${ }^{53}$ University of Notre Dame, Notre Dame, Indiana 46556, USA

${ }^{54}$ Ohio State University, Columbus, Ohio 43210, USA

${ }^{55}$ University of Oregon, Eugene, Oregon 97403, USA

${ }^{56}$ Università di Padova, Dipartimento di Fisica and INFN, I-35131 Padova, Italy

${ }^{57}$ Laboratoire de Physique Nucléaire et de Hautes Energies, IN2P3/CNRS, Université Pierre et Marie Curie-Paris6, Université Denis Diderot-Paris7, F-75252 Paris, France

${ }^{58}$ University of Pennsylvania, Philadelphia, Pennsylvania 19104, USA

${ }^{59}$ Università di Perugia, Dipartimento di Fisica and INFN, I-06100 Perugia, Italy

${ }^{60}$ Università di Pisa, Dipartimento di Fisica, Scuola Normale Superiore and INFN, I-56127 Pisa, Italy

${ }^{61}$ Prairie View A\&M University, Prairie View, Texas 77446, USA

${ }^{62}$ Princeton University, Princeton, New Jersey 08544, USA

${ }^{63}$ Università di Roma La Sapienza, Dipartimento di Fisica and INFN, I-00185 Roma, Italy

${ }^{64}$ Universität Rostock, D-18051 Rostock, Germany

${ }^{65}$ Rutherford Appleton Laboratory, Chilton, Didcot, Oxon, OX11 0QX, United Kingdom

${ }^{66}$ DSM/Dapnia, CEA/Saclay, F-91191 Gif-sur-Yvette, France

${ }^{67}$ University of South Carolina, Columbia, South Carolina 29208, USA

${ }^{68}$ Stanford Linear Accelerator Center, Stanford, California 94309, USA

${ }^{69}$ Stanford University, Stanford, California 94305-4060, USA

${ }^{70}$ State University of New York, Albany, New York 12222, USA

${ }^{71}$ University of Tennessee, Knoxville, Tennessee 37996, USA

${ }^{72}$ University of Texas at Austin, Austin, Texas 78712, USA

${ }^{73}$ University of Texas at Dallas, Richardson, Texas 75083, USA

${ }^{74}$ Università di Torino, Dipartimento di Fisica Sperimentale and INFN, I-10125 Torino, Italy

${ }^{75}$ Università di Trieste, Dipartimento di Fisica and INFN, I-34127 Trieste, Italy

${ }^{76}$ IFIC, Universitat de Valencia-CSIC, E-46071 Valencia, Spain

${ }^{77}$ University of Victoria, Victoria, British Columbia, Canada V8W $3 P 6$

${ }^{78}$ Department of Physics, University of Warwick, Coventry CV4 7AL, United Kingdom

${ }^{79}$ University of Wisconsin, Madison, Wisconsin 53706, USA

${ }^{80}$ Yale University, New Haven, Connecticut 06511, USA

(Received 18 July 2007; published 21 November 2007)

*Deceased.

${ }^{\dagger}$ Now at Tel Aviv University, Tel Aviv, 69978, Israel.

\#Also with Università di Perugia, Dipartimento di Fisica, Perugia, Italy.

${ }^{\S}$ Also with Università della Basilicata, Potenza, Italy.

"Also with Universitat de Barcelona, Facultat de Fisica, Departament ECM, E-08028 Barcelona, Spain. 
We present updated measurements of the branching fractions and $C P$ asymmetries for $B^{0} \rightarrow \pi^{0} \pi^{0}$, $B^{ \pm} \rightarrow \pi^{ \pm} \pi^{0}$, and $B^{ \pm} \rightarrow K^{ \pm} \pi^{0}$. Based on a sample of $383 \times 10^{6} Y(4 S) \rightarrow B \bar{B}$ decays collected by the $B A B A R$ detector at the PEP-II asymmetric-energy $B$ factory at SLAC, we measure $\mathcal{B}\left(B^{0} \rightarrow \pi^{0} \pi^{0}\right)=$ $(1.47 \pm 0.25 \pm 0.12) \times 10^{-6}, \mathcal{B}\left(B^{ \pm} \rightarrow \pi^{ \pm} \pi^{0}\right)=(5.02 \pm 0.46 \pm 0.29) \times 10^{-6}$, and $\mathcal{B}\left(B^{ \pm} \rightarrow K^{ \pm} \pi^{0}\right)=$ $(13.6 \pm 0.6 \pm 0.7) \times 10^{-6}$. We also measure the $C P$ asymmetries $\mathcal{C}_{\pi^{0} \pi^{0}}=-0.49 \pm 0.35 \pm 0.05$, $\mathcal{A}_{\pi^{ \pm} \pi^{0}}=0.03 \pm 0.08 \pm 0.01$, and $\mathcal{A}_{K^{ \pm} \pi^{0}}=0.030 \pm 0.039 \pm 0.010$. Finally, we present bounds on the Cabibbo-Kobayashi-Maskawa angle $\alpha$ using isospin relations.

In the standard model (SM) of particle physics, the charged-current couplings of the quark sector are described by the Cabibbo-Kobayashi-Maskawa (CKM) matrix elements $V_{\mathrm{qq}^{\prime}}$ [1]. The consistency of multiple measurements of the sides and angles of the CKM unitarity triangle provides a stringent test of the SM, and also provides constraints on non-SM physics. The CKM angle $\alpha \equiv$ $\arg \left[-\left(V_{\mathrm{td}} V_{\mathrm{tb}}^{*}\right) /\left(V_{\mathrm{ud}} V_{\mathrm{ub}}^{*}\right)\right]$ can be measured from the interference between $b \rightarrow u$ quark decays with and without $B^{0} \leftrightarrow \bar{B}^{0}$ mixing. In the limit of one (tree) amplitude, $\sin 2 \alpha$ can be extracted from the $C P$ asymmetries in $B^{0} \rightarrow$ $\pi^{+} \pi^{-}$decays [2]. However, the size of the branching fraction of $B^{0} \rightarrow \pi^{0} \pi^{0}$, relative to $B^{ \pm} \rightarrow \pi^{ \pm} \pi^{0}$ and $B^{0} \rightarrow$ $\pi^{+} \pi^{-}$, indicates that there is another significant (penguin) amplitude, with a different $C P$-violating (weak) phase, contributing to the decay. The deviation of the asymmetry obtained from $B \rightarrow \pi \pi$ decays, $\sin 2 \alpha_{\text {eff }}$, from $\sin 2 \alpha$ can be measured using the isospin-related decays $B^{ \pm} \rightarrow \pi^{ \pm} \pi^{0}$ and $B^{0} \rightarrow \pi^{0} \pi^{0}$ [3-5]. In the SM, the charge asymmetry is expected to be very small in the decay $B^{ \pm} \rightarrow \pi^{ \pm} \pi^{0}$ since penguin diagrams cannot contribute to the $I=2$ final state. However, a nonzero time-integrated $C P$ asymmetry in the decay $B^{0} \rightarrow \pi^{0} \pi^{0}$ is expected if penguin and tree amplitudes have different weak and $C P$-conserving (strong) phases.

The $B \rightarrow K \pi$ system also exhibits interesting $C P$-violating features, including direct $C P$ violation in $B^{0} \rightarrow K^{+} \pi^{-}$decays [6-8]. Sum rules derived from Uspin symmetry and parameters from the $B \rightarrow \pi \pi$ system relate the branching fraction and charge asymmetry of $B^{ \pm} \rightarrow K^{ \pm} \pi^{0}$ decays to other decays in the $K \pi$ system $[9,10]$. The $C P$ asymmetry in $B^{ \pm} \rightarrow K^{ \pm} \pi^{0}$ is expected to have the same sign and roughly the same magnitude as the $C P$ asymmetry in $B^{0} \rightarrow K^{+} \pi^{-}$in the absence of colorsuppressed tree and electroweak-penguin amplitudes.

Based on a sample of $383 \times 10^{6} Y(4 S) \rightarrow B \bar{B}$ decays, we report updated measurements of the branching fraction for $B^{0} \rightarrow \pi^{0} \pi^{0}$ and the time-integrated $C P$ asymmetry, $\mathcal{C}_{\pi^{0} \pi^{0}}$, defined as

$$
\mathcal{C}_{\pi^{0} \pi^{0}} \equiv \frac{\left|A_{00}\right|^{2}-\left|\bar{A}_{00}\right|^{2}}{\left|A_{00}\right|^{2}+\left|\bar{A}_{00}\right|^{2}}
$$

where $A_{00}\left(\bar{A}_{00}\right)$ is the $B^{0}\left(\bar{B}^{0}\right) \rightarrow \pi^{0} \pi^{0}$ decay amplitude. We also measure the branching fractions for $B^{ \pm} \rightarrow h^{ \pm} \pi^{0}$ $\left(h^{ \pm}=\pi^{ \pm}, K^{ \pm}\right)$and the corresponding charge asymme- tries

$$
\mathcal{A}_{h^{ \pm} \pi^{0}} \equiv \frac{N_{\pi^{-} \pi^{0}}-N_{\pi^{+} \pi^{0}}}{N_{\pi^{-} \pi^{0}}+N_{\pi^{+} \pi^{0}}},
$$

where $A_{ \pm 0}\left(\bar{A}_{ \pm 0}\right)$ is the $B^{+}\left(B^{-}\right)$decay amplitude.

The $B A B A R$ detector is described in Ref. [11]. Charged particle momenta are measured with a tracking system consisting of a five-layer silicon vertex tracker (SVT) and a 40-layer drift chamber (DCH) surrounded by a $1.5-\mathrm{T}$ solenoidal magnet. An electromagnetic calorimeter (EMC) comprising $6580 \mathrm{CsI}(\mathrm{Tl})$ crystals is used to measure photon energies and positions. The photon energy resolution in the EMC is $\sigma_{E} / E=\left\{2.3(\mathrm{GeV})^{1 / 4} / E^{1 / 4} \oplus\right.$ $1.9\} \%$, and the angular resolution from the interaction point is $\sigma_{\theta}=3.9^{\circ} / \sqrt{E / \mathrm{GeV}}$. Charged hadrons are identified with a detector of internally reflected Cherenkov light (DIRC) and ionization measurements in the tracking detectors. The average $K-\pi$ separation in the DIRC varies from $12 \sigma$ at a laboratory momentum of $1.5 \mathrm{GeV} / c$ to $2 \sigma$ at $4.5 \mathrm{GeV} / c$ [11].

For the reconstruction of $B^{ \pm} \rightarrow h^{ \pm} \pi^{0}$ events, we require the track from the $B$ candidate to have at least 12 hits in the DCH and be associated with at least 5 photons in the DIRC. The measured Cherenkov opening angle $\theta_{C}$ must be within $4 \sigma$ of the expectation for the pion or kaon hypothesis and $\theta_{C}$ must be greater than $10 \mathrm{mrad}$ from the proton hypothesis. Electrons are removed from the sample by vetoing candidates based on their energy loss in the SVT and $\mathrm{DCH}$ and a comparison of the track momentum and deposited energy in the EMC.

While $\pi^{0}$ meson candidates are mostly formed from two EMC clusters, we increase our $\pi^{0}$ efficiency compared to Ref. [4] by $\sim 10 \%$ by including $\pi^{0}$ candidates consisting of two overlapping photon clusters ("merged" $\pi^{0}$ ) and candidates with one photon cluster and two tracks consistent with being a photon conversion inside the detector. Photon conversions are selected from pairs of oppositely charged tracks with an invariant mass less than $30 \mathrm{MeV} / \mathrm{c}^{2}$, a vertex that lies within the detector, and a total momentum vector that points back to the beamspot. EMC clusters are required to have energies greater than $0.03 \mathrm{GeV}$ and a transverse shower shape consistent with a photon. To reduce the background from random photon combinations, the cosine of the angle between the direction of the decay photons in the center-of-mass system of the parent $\pi^{0}$ and the $\pi^{0}$ flight direction in the lab frame must be less than 
0.95. For candidates consisting of two EMC clusters or one cluster and a converted photon, the reconstructed $\pi^{0}$ mass is required to be between 110 and $160 \mathrm{MeV} / c^{2}$, and the candidates are then kinematically fit with their mass constrained to the $\pi^{0}$ mass. We distinguish merged $\pi^{0}$ candidates from single photons and other neutral hadrons using the second transverse moment, $S=\sum_{i} E_{i} \times\left(\Delta \alpha_{i}\right)^{2} / E$, where $E_{i}$ is the energy deposited in each CsI(Tl) crystal, and $\Delta \alpha_{i}$ is the angle between the cluster centroid and the crystal. Because merged $\pi^{0} \mathrm{~s}$ are caused by two overlapping photon clusters, they have a larger $S$ than solitary photons. We use a large sample of $\pi^{0}$ s from $\tau^{ \pm} \rightarrow \rho^{ \pm} \nu$ decays to validate that our Monte Carlo simulation (MC) accurately simulates merged $\pi^{0} \mathrm{~s}$ and photon conversions, as well as our overall $\pi^{0}$ efficiency.

We use two kinematic variables to isolate $B^{0} \rightarrow \pi^{0} \pi^{0}$ and $B^{ \pm} \rightarrow h^{ \pm} \pi^{0}$ candidates from the large background of $e^{+} e^{-} \rightarrow q \bar{q}(q=u, d, s, c)$ continuum events. The first is the beam-energy-substituted mass $\mathrm{m}_{\mathrm{ES}}=$ $\sqrt{\left(s / 2+\mathbf{p}_{i} \cdot \mathbf{p}_{B}\right)^{2} / E_{i}^{2}-\mathbf{p}_{B}^{2}}$, where $\sqrt{s}$ is the total $e^{+} e^{-}$ center-of-mass (CM) energy, $\left(E_{i}, \mathbf{p}_{i}\right)$ is the fourmomentum of the initial $e^{+} e^{-}$system, and $\mathbf{p}_{B}$ is the $B$-candidate momentum, both measured in the laboratory frame. The second variable is $\Delta E=E_{B}-\sqrt{s} / 2$, where $E_{B}$ is the $B$ candidate energy in the $\mathrm{CM}$ frame. For $B^{ \pm} \rightarrow$ $h^{ \pm} \pi^{0}$, we require $\mathrm{m}_{\mathrm{ES}}>5.22 \mathrm{GeV} / c^{2}$ and $-0.11 \mathrm{GeV}<$ $\Delta E<0.15 \mathrm{GeV}$. We define the main signal region in the $B^{0} \rightarrow \pi^{0} \pi^{0}$ analysis as $\mathrm{m}_{\mathrm{ES}}>5.20 \mathrm{GeV} / c^{2}$ and $|\Delta E|<$ $0.20 \mathrm{GeV}$.

To further discriminate the signal from $q \bar{q}$ backgrounds, we exploit the event topology variable $\theta_{S}$ : the angle in the $\mathrm{CM}$ frame between the sphericity axis of the $B$ candidate's decay products and that of the remaining neutral clusters and charged tracks in the rest of the event. Since the distribution of $\left|\cos \theta_{S}\right|$ peaks at 1 for $q \bar{q}$ events, we require $\left|\cos \theta_{S}\right|<0.8(0.7)$ for events with a $B^{ \pm} \rightarrow h^{ \pm} \pi^{0}\left(B^{0} \rightarrow\right.$ $\left.\pi^{0} \pi^{0}\right)$ candidate. To further improve background separation, we construct a Fisher discriminant $\mathcal{F}$ from the sums $\sum_{i} p_{i}$ and $\sum_{i} p_{i} \cos ^{2} \theta_{i}$, where $p_{i}$ is the $\mathrm{CM}$ momentum and $\theta_{i}$ is the angle with respect to the thrust axis of the $B$ candidate's daughters, in the CM frame, of all tracks and clusters not used to reconstruct the $B$ meson.

We use an extended, unbinned maximum likelihood (ML) fit to determine the number of signal events and the associated asymmetries. The probability density function (PDF) $\mathcal{P}_{i}\left(\vec{x}_{j} ; \vec{\alpha}_{i}\right)$ for event $j$ and signal or background hypothesis $i$ is the product of PDFs for the variables $\vec{x}_{j}$, given the set of parameters $\vec{\alpha}_{i}$. The likelihood function $\mathcal{L}$ is

$$
\mathcal{L}=\exp \left(-\sum_{i=1}^{M} n_{i}\right) \prod_{j=1}^{N}\left[\sum_{i=1}^{M} n_{i} \mathcal{P}_{i}\left(\vec{x}_{j} ; \vec{\alpha}_{i}\right)\right],
$$

where $N$ is the number of events, $n_{i}$ is the PDF coefficient for hypothesis $i$, and $M$ is the total number of signal and background hypotheses.

In the $B^{0} \rightarrow \pi^{0} \pi^{0}$ fit, the variables $\vec{x}_{j}$ are $m_{\mathrm{ES}}, \Delta E$, and $\mathcal{F}$. In addition to the signal and $q \bar{q}$ background, we expect background events from the charmless decays $B^{ \pm} \rightarrow$ $\rho^{ \pm} \pi^{0}$ and $B^{0} \rightarrow K_{S}^{0} \pi^{0}\left(K_{S}^{0} \rightarrow \pi^{0} \pi^{0}\right)$ to contribute $61 \pm 7$ events in the signal region, as determined from MC, so we include an additional component in the fit to account for this $B \bar{B}$ background. For the $B^{0} \rightarrow \pi^{0} \pi^{0}$ signal and the $B \bar{B}$ background, we observe a correlation coefficient between $m_{\mathrm{ES}}$ and $\Delta E$ of $\sim 0.2$, so a two-dimensional PDF, derived from MC simulation, is used to parametrize these distributions. The $q \bar{q}$ background PDF is described by an ARGUS threshold function [12] in $m_{\mathrm{ES}}$ and a polynomial in $\Delta E$. We divide the $\mathcal{F}$ distribution from signal MC into ten equally populated bins, and use a parametric step function to describe the distribution for all of the signal and background hypotheses. We fix the relative size of the $\mathcal{F}$ bins for the signal and $B \bar{B}$ background to values taken from MC. These values are verified with a sample of fully reconstructed $B$ meson decays. Continuum $\mathcal{F}$ parameters are free in the fit.

In order to measure the time-integrated $C P$ asymmetry $\mathcal{C}_{\pi^{0} \pi^{0}}$, we use the remaining tracks and clusters in a multivariate technique [13] to determine the flavor $\left(B^{0}\right.$ or $\left.\bar{B}^{0}\right)$ of the other $B$ meson in the event $\left(B_{\mathrm{tag}}\right)$. Events are assigned to one of seven mutually exclusive categories $k$ (including untagged events with no flavor information) based on the estimated mistag probability $w_{k}$ and on the source of the tagging information. The PDF coefficient for $B^{0} \rightarrow \pi^{0} \pi^{0}$ is given by

$$
n_{\pi^{0} \pi^{0}, k}=\frac{1}{2} f_{k} N_{\pi^{0} \pi^{0}}\left[1-s_{j}\left(1-2 \chi_{\mathrm{d}}\right)\left(1-2 w_{k}\right) \mathcal{C}_{\pi^{0} \pi^{0}}\right],
$$

where $N_{\pi^{0}} \pi^{0}$ is the total number of $B^{0} \rightarrow \pi^{0} \pi^{0}$ decays,

TABLE I. The results for the $B^{0} \rightarrow \pi^{0} \pi^{0}$ and $B^{ \pm} \rightarrow h^{ \pm} \pi^{0}$ decays. For each mode we show the number of signal events, $N_{S}$, number of continuum events, $N_{\text {cont }}$, number of $B$-background events, $N_{\text {Bbkg }}$, total detection efficiency, $\varepsilon$, branching fraction, $\mathcal{B}$, and asymmetry, $\mathcal{A}_{h^{ \pm} \pi^{0}}$ or $\mathcal{C}_{\pi^{0} \pi^{0}}$. Uncertainties are statistical for $N_{S}$ and $N_{\text {cont }}$, while for the branching fractions and asymmetries they are statistical and systematic, respectively.

\begin{tabular}{lrccccc}
\hline \hline Mode & \multicolumn{1}{c}{$N_{S}$} & $N_{\text {cont }}\left(10^{3}\right)$ & $N_{\text {Bbkg }}$ & $\varepsilon(\%)$ & $\mathcal{B}\left(10^{-6}\right)$ & Asymmetry \\
\hline$B^{0} \rightarrow \pi^{0} \pi^{0}$ & $154 \pm 27$ & $17.67 \pm 0.13$ & $61 \pm 7$ & 27.3 & $1.47 \pm 0.25 \pm 0.12$ & $-0.49 \pm 0.35 \pm 0.05$ \\
$B^{ \pm} \rightarrow \pi^{ \pm} \pi^{0}$ & $627 \pm 58$ & $58.75 \pm 0.24$ & $69 \pm 3$ & 32.5 & $5.02 \pm 0.46 \pm 0.29$ & $0.03 \pm 0.08 \pm 0.01$ \\
$B^{ \pm} \rightarrow K^{ \pm} \pi^{0}$ & $1364 \pm 57$ & $25.07 \pm 0.17$ & $9 \pm 2$ & 26.6 & $13.6 \pm 0.6 \pm 0.7$ & $0.030 \pm 0.039 \pm 0.010$ \\
\hline \hline
\end{tabular}


$\chi_{\mathrm{d}}=0.188 \pm 0.004[14]$ is the time-integrated mixing probability, and $s_{j}=+1(-1)$ when the $B_{\text {tag }}$ is a $B^{0}\left(\bar{B}^{0}\right)$. The fraction of events in each category, $f_{k}$, and the mistag rate are determined from a large sample of $B^{0} \rightarrow$ $D^{(*)}(n \pi) \pi$ decays.

For the $B^{ \pm} \rightarrow h^{ \pm} \pi^{0}$ fit, along with $m_{\mathrm{ES}}, \Delta E$, and $\mathcal{F}$, we include the Cherenkov angle $\theta_{C}$ to measure the $B^{ \pm} \rightarrow$ $\pi^{ \pm} \pi^{0}$ and $B^{ \pm} \rightarrow K^{ \pm} \pi^{0}$ yields and asymmetries simultaneously. The difference between the expected and measured Cherenkov angle, divided by the uncertainty, is described by two Gaussian distributions. The values for $m_{\mathrm{ES}}$ and $\Delta E$ are calculated assuming the track is a pion, so a $B^{ \pm} \rightarrow K^{ \pm} \pi^{0}$ event will have $\Delta E$ shifted by a value dependent on the track momentum, typically $-45 \mathrm{MeV}$. For the signal, the $m_{\mathrm{ES}}$ and $\Delta E$ distributions are modeled as Gaussian functions with low-side power-law tails. The means of these distributions and the $m_{\mathrm{ES}}$ width are determined in the fit, while the $\Delta E$ width is determined by $\mathrm{MC}$ simulation. We expect $69 \pm 3$ background events in the $B^{ \pm} \rightarrow \pi^{ \pm} \pi^{0}$ signal region from other $B$ meson decays, mainly from the same $B$ decays as in the $B^{0} \rightarrow \pi^{0} \pi^{0}$ case. For the $B^{ \pm} \rightarrow K^{ \pm} \pi^{0}$ signal region we expect $9 \pm 2$ events from $B \rightarrow X_{s} \gamma$ and $B^{0} \rightarrow \rho^{+} K^{-}$. The PDFs for the $B \bar{B}$ backgrounds, the $q \bar{q}$ background, and the signal $\mathcal{F}$ are all treated the same as in the $B^{0} \rightarrow \pi^{0} \pi^{0}$ case. The PDF coefficient for $B^{ \pm} \rightarrow h^{ \pm} \pi^{0}$ is given by $n_{i}=\frac{1}{2} N_{i}(1-$ $q_{j} \mathcal{A}_{i}$ ), where $\mathcal{A}_{i}$ is the charge asymmetry, and $q_{j}= \pm 1$ is the charge of the $B$ candidate.

The results from the $B^{0} \rightarrow \pi^{0} \pi^{0}$ and $B^{ \pm} \rightarrow h^{ \pm} \pi^{0} \mathrm{ML}$ fits are summarized in Table I. In a total of 17881 events, we find $154 \pm 27 B^{0} \rightarrow \pi^{0} \pi^{0}$ decays and an asymmetry $\mathcal{C}_{\pi^{0} \pi^{0}}=-0.49 \pm 0.35$. For the $B^{ \pm} \rightarrow h^{ \pm} \pi^{0}$ fit, we find $627 \pm 58 \quad B^{ \pm} \rightarrow \pi^{ \pm} \pi^{0}$ and $1364 \pm 57 \quad B^{ \pm} \rightarrow K^{ \pm} \pi^{0}$ events in a total of 85895 events. All of the correlations among the signal variables are less than 5\%. In Fig. 1 we use the event weighting and background subtraction method described in Ref. [15] to show signal and background distributions for $B^{0} \rightarrow \pi^{0} \pi^{0}$ events. Signal and background distributions for $B^{ \pm} \rightarrow h^{ \pm} \pi^{0}$ events are shown in Fig. 2 using the same method.

In order to account for a small bias in the $B^{ \pm} \rightarrow h^{ \pm} \pi^{0}$ asymmetries arising from the difference in the $\pi^{+}$and $\pi^{-}$reconstruction efficiencies and the $K^{+}$and $K^{-}$hadronic interaction cross sections in the $B A B A R$ detector, the
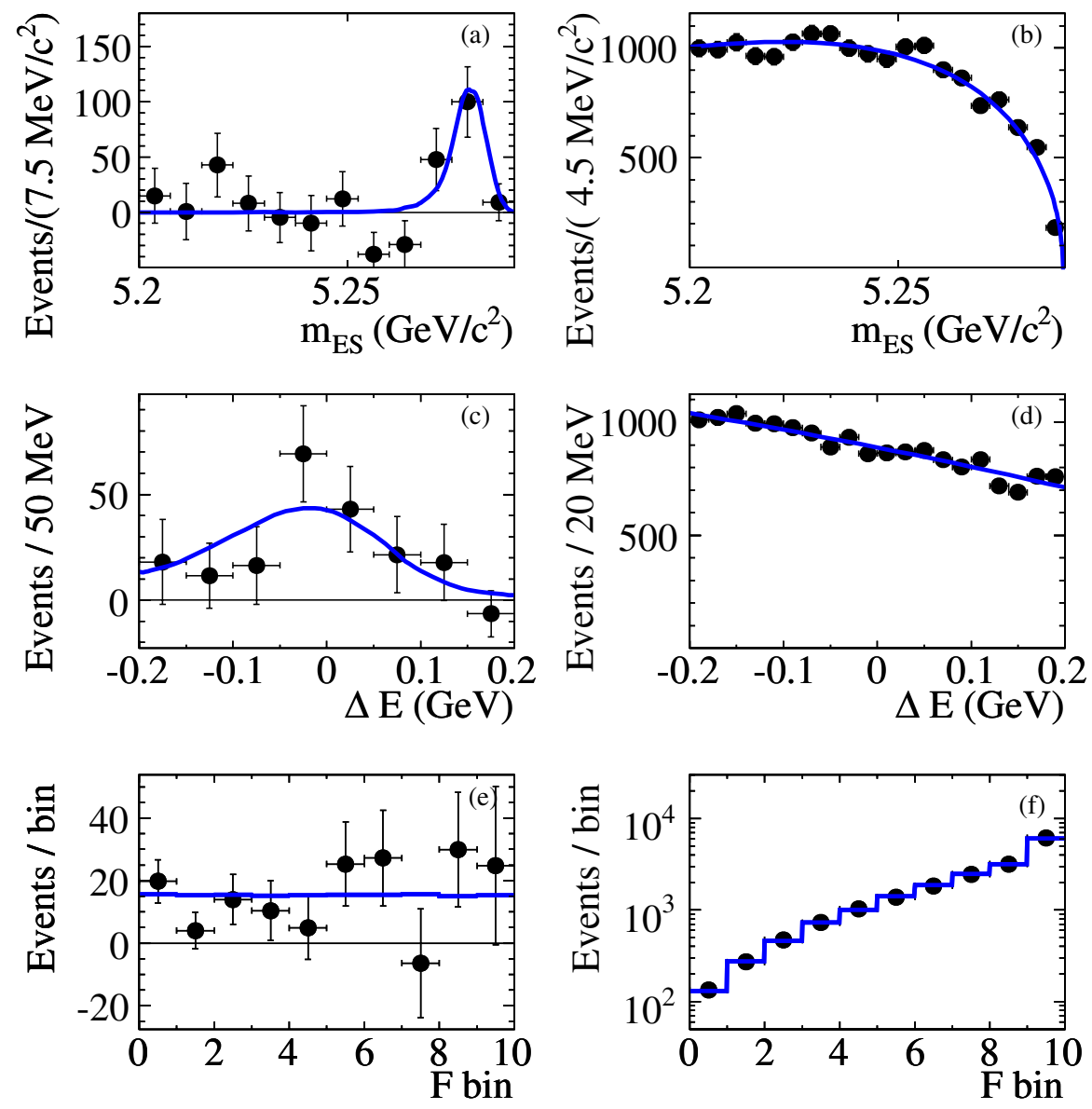

FIG. 1 (color online). Distributions made with the event weighting and background subtraction method described in Ref. [15] and PDF projections for the likelihood fit variables in the $B^{0} \rightarrow \pi^{0} \pi^{0}$ fit. Shown are $m_{\mathrm{ES}}[(\mathrm{a}),(\mathrm{b})], \Delta E[(\mathrm{c}),(\mathrm{d})]$, and $\mathcal{F}[(\mathrm{e}),(\mathrm{f})]$ for signal $[(\mathrm{a}),(\mathrm{c}),(\mathrm{e})]$ and continuum background [(b),(d),(f)]. 

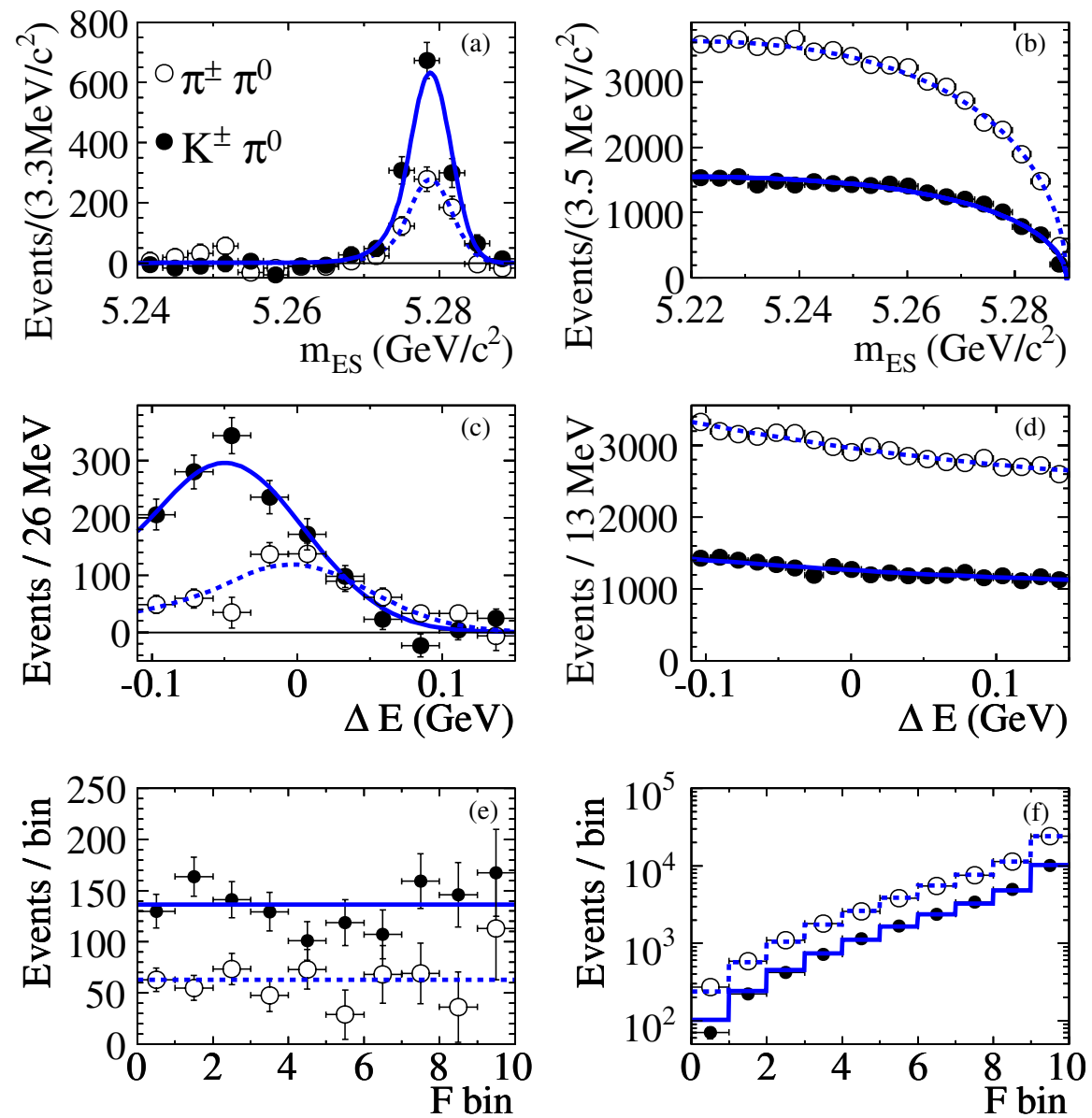

FIG. 2 (color online). Distributions made with the event weighting and background subtraction method described in Ref. [15] and PDF projections of the likelihood fit variables from the $B^{ \pm} \rightarrow h^{ \pm} \pi^{0}$ fit. Shown are $m_{\mathrm{ES}}[(\mathrm{a}),(\mathrm{b})], \Delta E[(\mathrm{c}),(\mathrm{d})]$, and $\mathcal{F}[(\mathrm{e}),(\mathrm{f})]$ distributions for signal [(a),(c),(e)] and continuum background [(b),(d),(f)]. PDF projections for the $B^{ \pm} \rightarrow K^{ \pm} \pi^{0}$ signal and background are shown as solid lines, while the PDF projections for the $B^{ \pm} \rightarrow \pi^{ \pm} \pi^{0}$ signal and background are shown as dashed lines. $B^{ \pm} \rightarrow \pi^{ \pm} \pi^{0}$ signal and background are shown as open circles while the $B^{ \pm} \rightarrow K^{ \pm} \pi^{0}$ is shown as solid circles.

$B^{ \pm} \rightarrow \pi^{ \pm} \pi^{0}$ asymmetry is corrected by $+0.005 \pm 0.004$ and the $B^{ \pm} \rightarrow K^{ \pm} \pi^{0}$ asymmetry is corrected by $+0.008 \pm$ 0.008 . We determine the $\pi^{ \pm} \pi^{0}$ bias from a study of $\tau^{ \pm} \rightarrow$ $\rho^{ \pm} \nu$ decays and verify it using the continuum background in data. For the $B^{ \pm} \rightarrow K^{ \pm} \pi^{0}$ charge asymmetry bias, we use the continuum background and combine the results of the $\pi^{ \pm} \pi^{0}$ asymmetry study and the $K^{ \pm} \pi^{\mp}$ asymmetry study in Ref. [6]. After the bias correction we find $\mathcal{A}_{\pi^{ \pm} \pi^{0}}=0.03 \pm 0.08$ and $\mathcal{A}_{K^{ \pm} \pi^{0}}=0.030 \pm 0.039$.

The systematic uncertainties on the branching fraction and asymmetry measurements are summarized in Tables II and III, respectively. The largest systematic errors for the $B^{0} \rightarrow \pi^{0} \pi^{0}$ and $B^{ \pm} \rightarrow h^{ \pm} \pi^{0}$ branching fractions are from uncertainties in the $\pi^{0}$ reconstruction efficiency, signal selection efficiencies, $\mathcal{F}$ parameters, and $B \bar{B}$ background yields. We evaluate these systematic errors using either control samples of $\pi^{0}$ mesons from $\tau^{ \pm} \rightarrow \rho^{ \pm} \nu$ decays and fully reconstructed $B$ meson decays from data or by varying fixed parameters and refitting. We simulate radiative effects using the PHOTOS simulation package [16] and assign a systematic error equal to the difference between PHOTOS and the scalar QED calculation in Ref. [17]. For the $B^{ \pm} \rightarrow h^{ \pm} \pi^{0}$ analysis, we also include as a systematic a small $(<2 \%)$ fit bias due to correlations among fit varia-

TABLE II. Systematic errors on the branching fractions for $B^{ \pm} \rightarrow \pi^{ \pm} \pi^{0}, B^{ \pm} \rightarrow K^{ \pm} \pi^{0}$, and $B^{0} \rightarrow \pi^{0} \pi^{0}$.

\begin{tabular}{lccc}
\hline \hline \multicolumn{1}{c}{ Source } & $\Delta \mathcal{B}\left(\pi^{ \pm} \pi^{0}\right)$ & $\Delta \mathcal{B}\left(K^{ \pm} \pi^{0}\right)$ & $\Delta \mathcal{B}\left(\pi^{0} \pi^{0}\right)$ \\
\hline$\pi^{0}$ efficiency & $3.0 \%$ & $3.0 \%$ & $6.0 \%$ \\
$m_{\text {ES and } \Delta E \text { PDF }}$ & $1.7 \%$ & $1.7 \%$ & $4.0 \%$ \\
Selection efficiency & $2.8 \%$ & $3.0 \%$ & $2.7 \%$ \\
$\mathcal{F}$ PDF & $2.5 \%$ & $0.7 \%$ & $1.7 \%$ \\
$B \bar{B}$ backgrounds & $0.2 \%$ & $<0.1 \%$ & $2.1 \%$ \\
PHOTOS & $1.9 \%$ & $1.1 \%$ & $\ldots$ \\
Fit bias & $1.7 \%$ & $1.2 \%$ & $\ldots$ \\
Luminosity & $1.1 \%$ & $1.1 \%$ & $1.1 \%$ \\
Tracking efficiency & $0.5 \%$ & $0.5 \%$ & $\ldots$ \\
Total & $5.8 \%$ & $5.0 \%$ & $8.2 \%$ \\
\hline \hline
\end{tabular}


TABLE III. A summary of the systematic errors on the asymmetries $\mathcal{A}_{\pi^{ \pm} \pi^{0}}, \mathcal{A}_{K^{ \pm} \pi^{0}}$, and $\mathcal{C}_{\pi^{0} \pi^{0}}$. All values are expressed in units of $10^{-2}$.

\begin{tabular}{lccc}
\hline \hline \multicolumn{1}{c}{ Source } & $\Delta\left(\mathcal{A}_{\pi^{ \pm} \pi^{0}}\right)$ & $\Delta\left(\mathcal{A}_{K^{ \pm} \pi^{0}}\right)$ & $\Delta\left(\mathcal{C}_{\pi^{0} \pi^{0}}\right)$ \\
\hline$B \bar{B}$ backgrounds & 0.2 & $<0.1$ & 3.4 \\
Tagging & $\ldots$ & $\ldots$ & 2.5 \\
Tag-side interference & $\ldots$ & $\ldots$ & 1.6 \\
PDF parameters & 0.8 & 0.6 & 0.9 \\
Detector asymmetry & 0.4 & 0.8 & $\ldots$ \\
Measured $\chi_{\mathrm{d}}$ error & $\ldots$ & $\ldots$ & 0.8 \\
Total & 0.9 & 1.0 & 4.7 \\
\hline \hline
\end{tabular}

bles. The largest systematic uncertainties in the measurement of $\mathcal{C}_{\pi^{0} \pi^{0}}$ are from the uncertainty on the $B$ background $C P$ content, tag-side interference, and the tagging fractions and asymmetry of $B_{\mathrm{tag}}$. The major contributions to the systematic error on $\mathcal{A}_{h^{ \pm} \pi^{0}}$ are from the detector charge asymmetry and the $\Delta E$ and $\mathcal{F}$ PDF parametrization.

We extract information on $\Delta \alpha \equiv \alpha_{\text {eff }}-\alpha$ and $\alpha$ using isospin relations [3] that relate the decay amplitudes of $B \rightarrow \pi \pi$ decays and measurements of the branching fraction and time-dependent $C P$ asymmetries in the decay $B^{0} \rightarrow \pi^{+} \pi^{-}$from $B A B A R$ [6,7]. For each of the six observable quantities required to calculate $\alpha$ [ $\mathcal{B}\left(B^{0} \rightarrow\right.$ $\left.\pi^{+} \pi^{-}\right), \quad \mathcal{B}\left(B^{ \pm} \rightarrow \pi^{ \pm} \pi^{0}\right), \quad \mathcal{B}\left(B^{0} \rightarrow \pi^{0} \pi^{0}\right), \quad \mathcal{S}_{\pi^{+} \pi^{-}}$, $\mathcal{C}_{\pi^{+} \pi^{-}}$, and $\mathcal{C}_{\pi^{0} \pi^{0}}$ ], we generate an ensemble of simulated experiments with uncorrelated Gaussian distributions where the width on each distribution is the sum in quadrature of the statistical and systematic errors of that measurement. Sets of generated experiments that result in an unphysical asymmetry or violate isospin are removed from the sample. Using the resulting distributions for $\Delta \alpha$ and $\alpha$, we calculate a confidence level (C.L.) for each solution and plot the maximum value of 1 minus C.L. of the various solutions in Fig. 3. One can further constrain $\alpha$ by using the fact that the penguin amplitude contribution to $B \rightarrow \pi \pi$ decays must be very large if $\alpha$ is near 0 or $\pi$. We obtain a bound on the magnitude of the penguin amplitude from the branching fraction of the penguin-dominated decay $B_{s} \rightarrow$ $K^{+} K^{-}$[18] by making the conservative assumption of $S U$ (3) breaking at less than $\sim 100 \%$ [19]. In Fig. 3 we also show bounds on $\alpha$ when the size of the penguin amplitude is constrained by this assumption.

In summary, we measure the branching fractions and $C P$ asymmetries in $B^{0} \rightarrow \pi^{0} \pi^{0}, B^{ \pm} \rightarrow \pi^{ \pm} \pi^{0}$, and $B^{ \pm} \rightarrow$ $K^{ \pm} \pi^{0}$ decays reconstructed from a sample of approximately $383 \times 10^{6} B \bar{B}$ pairs. While all of these measurements represent significant improvements of the previous $B A B A R$ measurements, the error on $\mathcal{C}_{\pi^{0} \pi^{0}}$ shows the largest improvement. All results are consistent with previously published results from $B A B A R$ [4] and the Belle experiment [5], and supersede the previous $B A B A R$ results. For the $B \rightarrow \pi \pi$ decays, we find $\mathcal{B}\left(B^{0} \rightarrow \pi^{0} \pi^{0}\right)=(1.47 \pm$
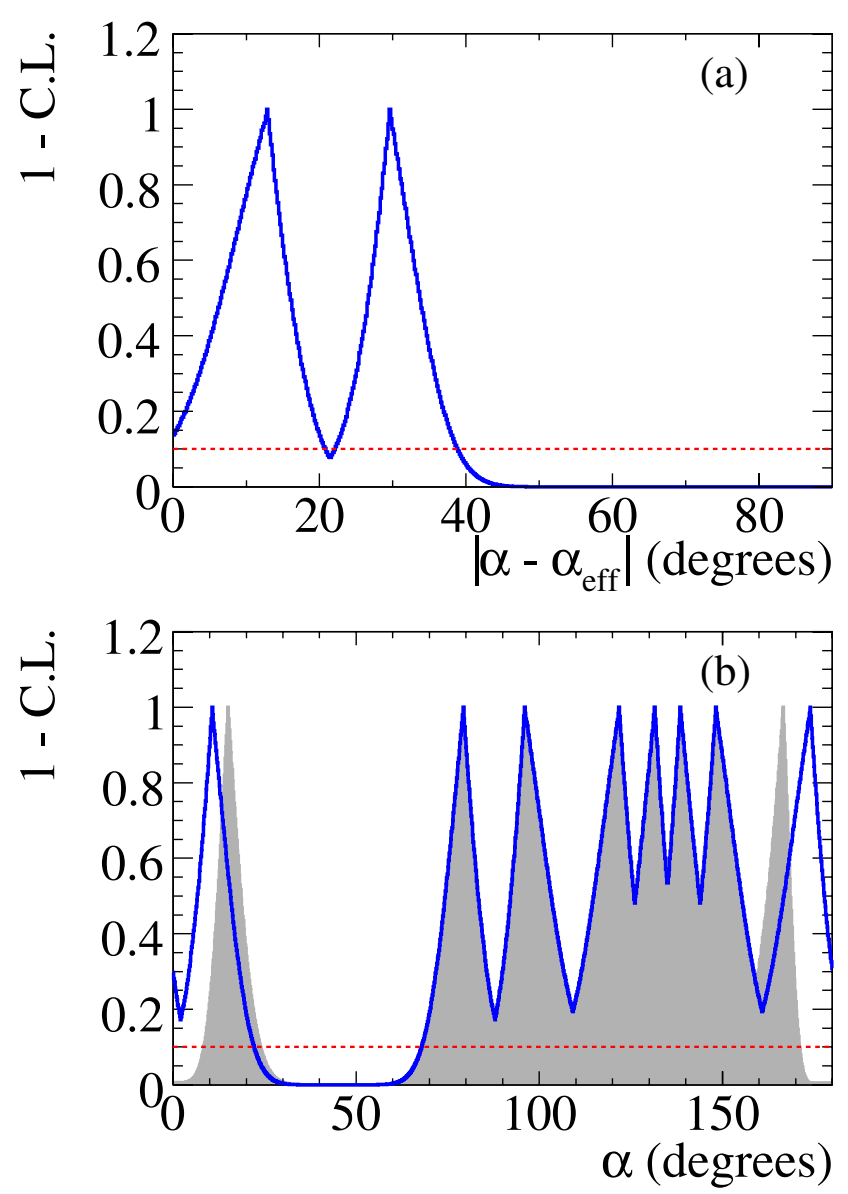

FIG. 3 (color online). Constraints on (a) the angle $\Delta \alpha=\mid \alpha-$ $\alpha_{\text {eff }} \mid$ and (b) $\alpha$ expressed as one minus the confidence level as a function of angle. We find an upper bound on $\Delta \alpha$ of $39^{\circ}$ at the $90 \%$ confidence level. In (b) the curve shows the bounds on $\alpha$ using the isospin method alone, while the shaded region shows the result with the $S U(3)$ requirement as discussed in the text.

$0.25 \pm 0.12) \times 10^{-6}, \mathcal{B}\left(B^{ \pm} \rightarrow \pi^{ \pm} \pi^{0}\right)=(5.02 \pm 0.46 \pm$ $0.29) \times 10^{-6}, \quad \mathcal{C}_{\pi^{0} \pi^{0}}=-0.49 \pm 0.35 \pm 0.05, \quad$ and $\mathcal{A}_{\pi^{ \pm} \pi^{0}}=0.03 \pm 0.08 \pm 0.01$. We constrain $\Delta \alpha$ to be less than $39^{\circ}$ and exclude the range $\left[25^{\circ}, 66^{\circ}\right]$ in $\alpha$ at $90 \%$ confidence level. If we consider only the preferred solution [20], we find $\alpha=\left(96_{-6}^{+10}\right)^{\circ}$. For the $B^{ \pm} \rightarrow K^{ \pm} \pi^{0}$ decay, we find $\mathcal{B}\left(B^{ \pm} \rightarrow K^{ \pm} \pi^{0}\right)=(13.6 \pm 0.6 \pm 0.7) \times$ $10^{-6}$ and $\mathcal{A}_{K^{ \pm} \pi^{0}}=0.030 \pm 0.039 \pm 0.010$. The difference between $\mathcal{A}_{K^{ \pm} \pi^{0}}$ and $\mathcal{A}_{K^{ \pm} \pi^{\mp}}=-0.107 \pm 0.019$ [6] indicates that the effect of color-suppressed tree and electroweak-penguin amplitudes are significant.

We are grateful for the excellent luminosity and machine conditions provided by our PEP-II colleagues, and for the substantial dedicated effort from the computing organizations that support BABAR. The collaborating institutions wish to thank SLAC for its support and kind hospitality. This work is supported by DOE and NSF (U.S.A.), NSERC (Canada), CEA and CNRS-IN2P3 (France), BMBF and 
STUDY OF $B^{0} \rightarrow \pi^{0} \pi^{0}, \ldots$

DFG (Germany), INFN (Italy), FOM (The Netherlands), NFR (Norway), MIST (Russia), MEC (Spain), and STFC (United Kingdom). Individuals have received support from
PHYSICAL REVIEW D 76, 091102(R) (2007)

the Marie Curie EIF (European Union) and the A. P. Sloan Foundation.
[1] N. Cabibbo, Phys. Rev. Lett. 10, 531 (1963); M. Kobayashi and T. Maskawa, Prog. Theor. Phys. 49, 652 (1973).

[2] Unless specifically stated, conjugate decay modes are assumed throughout this paper.

[3] M. Gronau and D. London, Phys. Rev. Lett. 65, 3381 (1990).

[4] B. Aubert et al. (BABAR Collaboration), Phys. Rev. Lett. 91, 241801 (2003); 91, 021801 (2003); 94, 181802 (2005).

[5] K. Abe et al. (Belle Collaboration), Phys. Rev. Lett. 94, 181803 (2005); Y. Chao et al. (Belle Collaboration), Phys. Rev. D 71, 031502 (2005); H. Ishino et al. (Belle Collaboration), Phys. Rev. Lett. 98, 211801 (2007); S.-W. Lin et al. (Belle Collaboration), Phys. Rev. Lett. 99, 121601 (2007).

[6] B. Aubert et al. (BABAR Collaboration), Phys. Rev. Lett. 99, 021603 (2007).

[7] B. Aubert et al. (BABAR Collaboration), Phys. Rev. D 75, 012008 (2007).

[8] Y. Chao et al. (Belle Collaboration), Phys. Rev. Lett. 93, 191802 (2004).

[9] M. Gronau and J.L. Rosner, Phys. Rev. D 71, 074019 (2005); M. Gronau, Phys. Lett. B 627, 82 (2005); M.
Gronau and J.L. Rosner, Phys. Rev. D 59, 113002 (1999); H. J. Lipkin, Phys. Lett. B 445, 403 (1999).

[10] A. Buras et al., Phys. Rev. Lett. 92, 101804 (2004); Nucl. Phys. B697, 133 (2004).

[11] B. Aubert et al. (BABAR Collaboration), Nucl. Instrum. Methods Phys. Res., Sect. A 479, 1 (2002).

[12] The function is $f(x) \propto x \sqrt{1-x^{2}} \exp \left[-\zeta\left(1-x^{2}\right)\right]$, where $x=m_{\mathrm{ES}} / m_{0}, m_{0}$ is the $m_{\mathrm{ES}}$ endpoint, and $\zeta$ the shape parameter.

[13] B. Aubert et al. (BABAR Collaboration), Phys. Rev. Lett. 94, 161803 (2005).

[14] W.-M. Yao et al. (Particle Data Group), J. Phys. G 33, 1 (2006).

[15] M. Pivk and F. R. Le Diberder, Nucl. Instrum. Methods Phys. Res., Sect. A 555, 356 (2005).

[16] E. Barberio and Z. Was, Comput. Phys. Commun. 79, 291 (1994).

[17] E. Baracchini and G. Isidori, Phys. Lett. B 633, 309 (2006).

[18] M. Morello et al. (CDF Collaboration), Nucl. Phys. B, Proc. Suppl. 170, 39 (2007).

[19] M. Bona et al. (UTfit Collaboration), Phys. Rev. D 76, 014015 (2007).

[20] M. Gronau and J. L. Rosner, Phys. Lett. B 651, 166 (2007). 